\title{
Functional domains of CCN1 (Cyr61) regulate breast cancer progression
}

\author{
JAMES O'KELLY ${ }^{1,5}$, ALICE CHUNG ${ }^{1}$, NATHAN LEMP ${ }^{1}$, KATYA CHUMAKOVA ${ }^{1}$, DONG YIN ${ }^{1}$, \\ HE-JING WANG ${ }^{3}$, JONATHAN SAID ${ }^{4}$, DORINA GUI ${ }^{4}$, CARL W. MILLER ${ }^{1}$, \\ BETH Y. KARLAN ${ }^{2}$ and H. PHILLIP KOEFFLER ${ }^{1}$ \\ ${ }^{1}$ Division of Hematology/Oncology, ${ }^{2}$ Department of Obstetrics and Gynecology, Cedars-Sinai Medical Center, \\ Departments of ${ }^{3}$ Biomathematics, and ${ }^{4}$ Pathology, UCLA School of Medicine, Los Angeles, CA, USA
}

Received January 28, 2008; Accepted March 21, 2008

\begin{abstract}
CCN1 plays diverse roles in cellular proliferation, survival, migration and angiogenesis. We determined the relationship between $\mathrm{CCN} 1$ protein expression and clinical factors that are important for the classification of breast cancer. CCN1 contains four functional domains; the contribution of each of the structural domains to the biological properties of CCN1 in breast cancer was investigated. We performed immunohistochemistry for $\mathrm{CCN} 1$ on a breast cancer tissue array, and conducted a detailed statistical analysis on the relationship between $\mathrm{CCN} 1$ protein expression and clinical factors that are important for the classification of breast cancer. The structure-function relationship was examined using four mutant constructs in which one of the modules (DM1-DM4) had been deleted. MCF-7 breast cancer cells were stably transfected with these constructs and their biological activity was tested in comparison to full-length $\mathrm{CCN} 1$. Staining of CCN1 in tumors was positively correlated with AJCC disease stage. A strong association also was found between lymph node involvement and high CCN1 expression in patients with invasive breast cancer; there was a significant increase in the breast cancer expression of CCN1 in patients with positive lymph nodes $(\mathrm{P}=0.004)$, and the levels of CCN1 correlated with the number of positive lymph nodes $(\mathrm{P}=0.0006)$. Deletion of module 4 rendered $\mathrm{CCN} 1$ unable to either bind heparin or associate with the extracellular matrix. Furthermore, MCF-7/DM4 cells demonstrated reduced cell spreading, migration and proliferation, indicating that module 4 of the protein is important for its ability to promote these activities. These findings indicate that CCN1 is involved throughout the clinical progression of breast cancer to an invasive phenotype. The multimodular structure
\end{abstract}

Correspondence to: Dr James O'Kelly, ${ }^{5}$ Present address: Global Medical Writing, Amgen, One Amgen Center Drive, MS 27-4-A, Thousand Oaks, CA 91320-1799, USA

E-mail: james.okelly@gmail.com

Key words: Cyr61, breast cancer, extracellular matrix of CCN1 enables it to fulfill multiple functions that may contribute to the different stages of cancer development, raising the prospect that specific regions of CCN1 could be targeted for therapeutic benefit to inhibit particular aspects of malignancy in breast cancer.

\section{Introduction}

The CCN1 gene encodes a matricellular protein that belongs to the CCN family $(1,2)$, comprising CCN1/Cyr61; CCN2/ CTGF (connective tissue growth factor); $\mathrm{CCN} 3 / \mathrm{NOV}$ (nephroblastoma overexpressed); and CCN4-6/WISP1-3 (Wntinducible secreted proteins). $\mathrm{CCN} 1$ plays diverse roles in cellular proliferation, survival, differentiation, adhesion and migration, as well as regulation of chemotaxis and angiogenesis $(3,4)$. Dysregulation of these processes is a common feature of tumorigenesis and cancer progression, associated with inappropriate expression of $\mathrm{CCN} 1$ in a variety of cancers. $\mathrm{CCN} 1$ has a complex role in tumorigenesis, demonstrating divergent functions and expression profiles in cancers from different tissues (5). For example, studies in our laboratory have detected high expression of CCN1 in breast cancers that were associated with increased tumorigenicity, but low expression in lung cancers where it was found to reduce cellular proliferation $(6,7)$. The wide-ranging and often conflicting effects of CCN1 and other family members have been attributed in part to their multimodular structure, which enables different regions of the protein to fulfill distinct functions (8). In this regard, an N-terminal cleavage product of CCN1 produced by breast cancer cells has been found to stimulate endothelial cell proliferation (9).

CCNs are a conserved family of proteins containing an $\mathrm{N}$-terminal signal peptide, followed by up to four functional domains that each share sequence similarities with other protein motifs (10). Thus, module 1 is termed the insulin-like growth factor binding (IGFBP) domain, module 2 the von Willebrand factor type C (VWC) domain, module 3 the thrombospondin type 1 (TSP) domain and module 4 contains the C-terminal growth factor cysteine knot (CT) domain. Each of the four modules is thought to act both independently and interdependently (8). Supporting this hypothesis are observations that binding partners of $\mathrm{CCN} 1$ interact with 
sites in different regions of the protein, such that module 2 contains binding sites for integrin $\alpha_{v} \beta_{3}(11)$, module 3 binds integrin $\alpha_{6} \beta_{1}(12)$, and binding sites for $\alpha_{6} \beta_{1}$, heparan sulfate proteoglycans (HSPGs) and integrin $\alpha_{M} \beta_{2}$ are located in module $4(13,14)$.

Integrin activation has been shown to play an important role in the development and progression of breast cancer, achieved through intracellular signaling via activation of downstream kinases such as ILK and MAPK (15-17). Overexpression of CCN1 in MCF-7 breast cancer cells leads to up-regulation of MAPK and $\mathrm{NF \kappa B}$ signaling in an integrin $\alpha_{v} \beta_{3}$-dependent manner, promoting cell survival and chemoresistance $(18,19)$. The diverse functions of CCN1 suggest that it may play a role at multiple stages of breast cancer, and elevated expression of CCN1 mRNA expression has been demonstrated in highly aggressive breast cancer cell lines and in samples from patients with more advanced stages of malignancy $(6,20-22)$. Overexpression of CCN1 in breast cancer cell lines promotes their cell growth, migration and in vivo angiogenesis $(6,23)$. Furthermore, CCN1 is thought to be an essential mediator of estrogen and progesteronestimulated growth of breast cancer cells $(21,24)$.

Previous studies have monitored transcript levels to examine the relationship between CCN1 expression and clinical factors $(22,25)$. The present study uses immunohistochemistry to determine protein levels of CCN1 in a tissue array of 168 patient samples. We describe a strong relationship between CCN1 protein expression and progression of breast cancer to lymph nodes involvement, which is associated with a worse prognosis. Furthermore, by expressing deletion mutants of CCN1 in MCF-7 cells, we have been able to show that the CT domain is important for the ability of the protein to promote migration and proliferation of breast cancer cells. These findings provide novel information on the functionality of the different modular domains of CCN1, and offer the possibility that targeting specific regions of CCN1 may have therapeutic implications for the treatment of breast cancer.

\section{Materials and methods}

Patient samples and immunohistochemistry. Tissue microarrays (TMAs) were obtained from the Cooperative Breast Cancer Tissue Resource (CBCTR) at the National Cancer Institute (NCI) (http://cbctr.nci.nih.gov/tma.html). TMA blocks consist of 288 cores taken from paraffin-embedded specimens that represent 252 breast cancer and normal breast specimens, plus 36 controls consisting of cell lines and normal non-breast tissue. The 252 normal breast and breast cancer cores include: 64 from node-negative breast cancer, 64 from node-positive breast cancer, 64 from metastatic breast cancer, 20 from DCIS and 20 from normal breast tissue.

Immunohistochemical staining for CCN1 was performed using a polyclonal antibody (H-78, Santa Cruz Biotechnology, Santa Cruz, CA). Heat-induced epitope retrieval was done with a pressure cooker and Tris buffer $(\mathrm{pH} 9.0)$ for 2 min. Staining was visualized with Dako Envision (Dako, Carpinteria, CA) conjugated to horseradish peroxidase followed by the diaminobenzidine reaction. Negative controls consisted of substitution of the primary antiserum with normal rabbit serum at the same dilution. Staining intensity was quantified using either integrated optical density or microscopic examination. Integrated optical density was quantified using Metamorph software (Universal Imaging) from images captured using a dual Xeon computer (Windows $\mathrm{XP}$ ) and an Insight (Spot) 4-megapixel color CCD camera. Microscopic examination was performed by 2 pathologists and all samples were blinded. The staining intensity was scored on a scale of negative to strong as follows: $0=0 \%$ of reactive cells with the score of $0 ; 1,1-25 \%$ of reactive cells with score $1 ; 2,26-50 \%$ of reactive cells with score 2 ; and $3,>50 \%$ of reactive cells with score 3 .

Statistical analysis. Specimens from which it was not possible to obtain data for the integrated OD measurements were excluded from the analysis. Spearman correlation coefficients were used to correlate the levels of $\mathrm{CCN} 1$ with the tumor size, American Joint Committee on Cancer (AJCC) stage, number of positive lymph nodes and age of the patients. The ER status, PR status, T-, N- and M-stage were compared using t-tests and Wilcoxon rank sum tests. A P-value of $<0.05$ was considered significant.

Cell culture. The breast cancer cell line MCF-7 was obtained from the American Type Tissue Culture Collection (Rockville, MD). Cells were cultured in Dulbecco's modified Eagle's medium (DMEM) (Life Technologies, Inc.) supplemented with $10 \%$ fetal calf serum (Gemini Bio-Products, Calabasas, CA), 10 units/ml penicillin-streptomycin (Life Technologies) and incubated at $37^{\circ} \mathrm{C}$ in $5 \% \mathrm{CO}_{2}$. Deletion mutants of $\mathrm{CCN} 1$ were generated by PCR amplification using full-length CCN1 cDNA as a template. Fragments were cloned into pcDNA3.1 vector (Invitrogen) and MCF-7 cells were stably transfected with the constructs as described previously (6).

Cell proliferation assay. Cells (100 $\mu \mathrm{l})$ were placed into 96well plates at $1 \times 10^{3}$ cells per well. After incubation for $96 \mathrm{~h}$, cell number was assessed by the MTT assay (Sigma). MTT is a water-soluble yellow salt that is catalyzed to an insoluble purple formazan by active mitochondrial enzymes of viable cells. The amount of purple crystals converted from MTT is proportional to the number of viable cells. MTT solution $(10 \mu \mathrm{l})(5 \mathrm{mg} / \mathrm{ml}$ in PBS) was added to each well for $4 \mathrm{~h}$, then all media were carefully aspirated from the crystals. DMSO $(100 \mu 1)$ was added to each well and plates were read at $540 \mathrm{~nm}$ with a plate reader.

Adhesion and cell spreading assay. The ECM proteins, collagen (type I), fibronectin and vitronectin were obtained from Sigma and diluted to a concentration of $50 \mu \mathrm{g} / \mathrm{ml}$ in PBS for use in adhesion and cell spreading assays. Wells of 96-well plates were coated with $35 \mu 1$ of solution overnight at $4^{\circ} \mathrm{C}$. Subsequently, wells were washed with PBS and blocked with $2.5 \%$ BSA in PBS for $2 \mathrm{~h}$ at room temperature. Cells cultured overnight in serum-free DMEM were detached from the culture dish by incubation with PBS containing $50 \mathrm{mM}$ EDTA. Cells were resuspended in serum-free media with $0.5 \%$ bovine serum albumin and added to wells at a concentration $1 \times 10^{4}$ cells/well. After $30 \mathrm{~min}$ unattached cells were aspirated, and attached cells were washed twice with PBS and stained 
with $0.25 \%$ crystal violet in $50 \%$ methanol for $15 \mathrm{~min}$. Stained cells were photographed and dissolved in DMSO for quantitation in an ELISA plate reader at OD $540 \mathrm{~nm}$.

Wounding and Boyden chamber assays for measurement of cell migration. For wounding assays, cells were grown to confluence and serum-starved overnight. Cells were cut with a sterile razor and images were captured at a fixed point along the cut surface. Boyden chambers (Nunc, Rochester $\mathrm{NY}$ ) with $8 \mu \mathrm{m}$ pore size were coated on the underside of the membrane with $50 \mu \mathrm{g} / \mathrm{ml}$ collagen for $1 \mathrm{~h}$ at room temperature. Cells were prepared as described for adhesion assays, and $2 \times 10^{5}$ cells in $300 \mu 1$ serum-free media were added to the top of each chamber with the lower chamber containing $300 \mu 1$ of serum-free medium plus $0.5 \%$ BSA. Chambers were placed in 24-well plates, and cells were allowed to migrate to the underside of the top chamber for $12 \mathrm{~h}$. The migratory cells that attached to the bottom surface of the membrane were stained with $0.25 \%$ crystal violet in $50 \%$ methanol for $15 \mathrm{~min}$. The stained cells were extracted with $10 \%$ acetic acid. The number of migratory cells per membrane was determined by absorbance at $550 \mathrm{~nm}$.

Western blotting and in vitro kinase assay. Protein from cells harvested for Western blotting was extracted with RIPA buffer (1\% NP-40, $0.5 \%$ sodium deoxycholate, $0.1 \%$ SDS, $50 \mathrm{mM}$ Tris-HCl, pH 7.5) containing protease inhibitor cocktail (Roche Molecular Biochemicals). Protein lysates $(40 \mu \mathrm{g})$ were boiled in Laemmli sample buffer (Bio-Rad), resolved by electrophoresis on 4-15\% SDS-polyacrylamide gels and transferred to PVDF membranes. Membranes were probed with antibodies (Santa Cruz) and developed using the enhanced chemiluminescence kit (Pierce). For heparin-pull down experiments, cells were lysed in IP Buffer $(50 \mathrm{mM}$ Tris $\mathrm{pH} 7.4,150 \mathrm{mM} \mathrm{NaCl}, 0.5 \% \mathrm{NP}-40)$, and $500 \mu \mathrm{g}$ of protein was incubated with rotation at $4^{\circ} \mathrm{C}$ overnight with heparin-agarose beads (Sigma). Beads were washed 3 times in PBS, boiled in Laemmli sample buffer and subject to Western blotting as described above. To extract proteins from the ECM, cells were first detached from the culture dish with PBS containing $50 \mathrm{mM}$ EDTA. Dishes were then washed with PBS and the ECM left behind was extracted by incubating with Laemmli sample buffer at $90^{\circ} \mathrm{C}$ for $5 \mathrm{~min}$. For in vitro kinase assays, immunoprecipitated proteins on beads were washed twice with lysis buffer and twice with kinase buffer (50 mM HEPES pH 7.0, $10 \mathrm{mM} \mathrm{MgCl}_{2}, 5 \mathrm{mM}$ $\mathrm{MnCl}_{2}, 1 \mathrm{mM}$ DTT). The beads were resuspended in $40 \mu \mathrm{l}$ of kinase buffer containing $2 \mu \mathrm{g}$ of myelin basic protein, $10 \mathrm{mM}$ ATP and $5 \mathrm{mCi}\left[\gamma^{-32} \mathrm{P}\right]-\mathrm{ATP}$. Labeled substrate was separated from free $\left[\gamma_{-}{ }^{32} \mathrm{P}\right]-\mathrm{ATP}$ by $4-15 \%$ SDS-polyacrylamide gel electrophoresis. The gel was dried and exposed to X-ray film.

Immunofluoresence. Cells grown on coverslips were fixed in $4 \%$ paraformaldehyde, washed three times with PBS, and subsequently permeabalized in blocking buffer $(0.6 \%$ Tween, $1 \%$ bovine serum albumin in PBS) for $20 \mathrm{~min}$ at room temperature. Coverslips were incubated with $1 \mu \mathrm{g} / \mathrm{ml}$ primary antibody in blocking buffer overnight at $4^{\circ} \mathrm{C}$, then washed with PBS and incubated with biotinylated secondary (a)

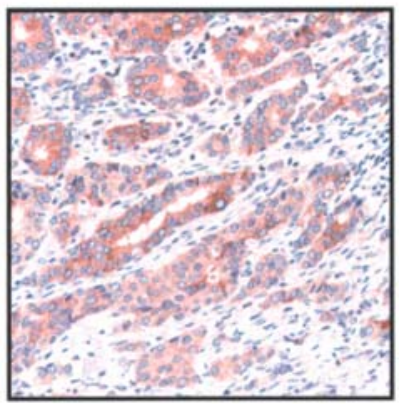

(b)

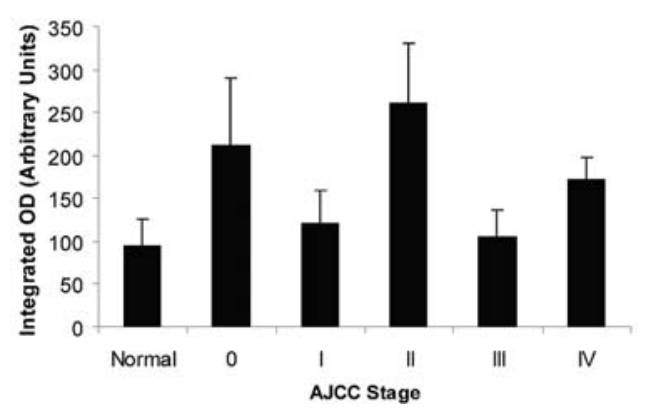

(c)

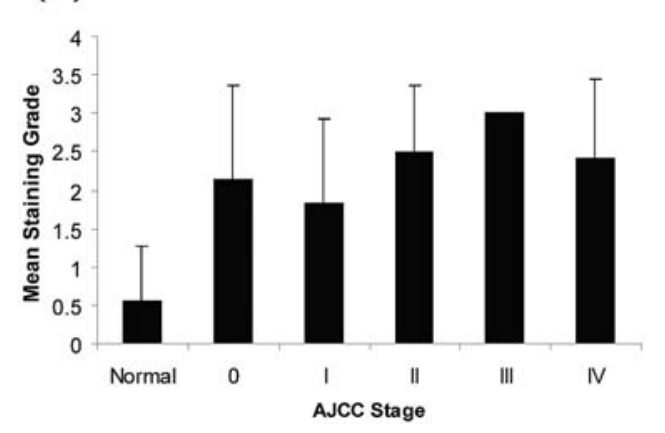

Figure 1. CCN1 protein overexpression in breast cancer patient samples. Immunohistochemical staining using an antibody against CCN1 protein was performed on a tissue array using samples from normal breast and breast cancer patients. (a) Examples of typical staining patterns observed. Strong CCN1 staining is located in the cytoplasm of the tumor epithelial cells. Staining levels of CCN1 were increased at different AJCC stages of disease in comparison to normal. Staining intensity was assessed using either IOD measurements (b) or by grading performed by a pathologist (c). Results represent the mean $\pm \mathrm{SD}$ of measurements taken from 9 normal, 8 stage 0,37 stage I, 63 stage II, 6 stage III and 54 stage IV samples.

antibody (Dako) in blocking buffer for $30 \mathrm{~min}$. The coverslips were washed with PBS and incubated with a $5 \%$ solution of RPE-avidin (Dako) before being mounted, and staining was visualized with a fluorescent microscope.

\section{Results}

CCN1 protein expression in breast tumors and relationship with clinical factors. Previous studies have demonstrated increased CCN1 mRNA expression in breast cancer compared to normal breast tissue. We performed a detailed statistical analysis to analyze carefully the relationship between CCN1 protein expression and clinical factors that are important for the classification of breast cancer. Tissue arrays containing samples from breast cancer and normal breast were immuno- 
Table I. Relationship between clinical features and Cyr61 staining.

\begin{tabular}{|c|c|c|c|c|c|}
\hline \multirow[t]{2}{*}{ Factors } & \multirow[t]{2}{*}{ IOD } & \multicolumn{4}{|c|}{ Grade } \\
\hline & & 0 & 1 & 2 & 3 \\
\hline \multicolumn{6}{|l|}{ ER } \\
\hline Positive $(n$, mean \pm SD) & $91,179 \pm 377$ & 9 & 9 & 17 & 61 \\
\hline Negative $(n$, mean $\pm S D)$ & $51,216 \pm 393$ & 7 & 3 & 12 & 31 \\
\hline Wilcoxon rank sum test & $\mathrm{P}=0.4378$ & \multicolumn{4}{|c|}{$\mathrm{P}=0.5756$} \\
\hline \multicolumn{6}{|l|}{ PR } \\
\hline Positive $(n$, mean $\pm S D)$ & $45,213 \pm 502$ & 8 & 2 & 10 & 29 \\
\hline Negative $(n$, mean $\pm S D)$ & $90,191 \pm 324$ & 8 & 9 & 18 & 59 \\
\hline Wilcoxon rank sum test & $\mathrm{P}=0.3510$ & \multicolumn{4}{|c|}{$\mathrm{P}=0.5750$} \\
\hline Tumor Size & 0.27 & \multicolumn{4}{|c|}{0.29} \\
\hline Spearman corr. coefficient & $\mathrm{P}=0.0009$ & \multicolumn{4}{|c|}{$\mathrm{P}=0.0003$} \\
\hline \multicolumn{6}{|l|}{ T-Stage } \\
\hline $\mathrm{T} 1$ & $78,128 \pm 198$ & 13 & 8 & 22 & 41 \\
\hline$\geq \mathrm{T} 2$ & $65,267 \pm 514$ & 4 & 4 & 8 & 51 \\
\hline Wilcoxon rank sum test & $\mathrm{P}=0.0001$ & \multicolumn{4}{|c|}{$\mathrm{P}=0.0011$} \\
\hline \multicolumn{6}{|l|}{ Node Status } \\
\hline Negative $(n$, mean $\pm S D)$ & $48,134 \pm 224$ & 8 & 4 & 15 & 24 \\
\hline Positive $(n$, mean \pm SD) & $66,270 \pm 509$ & 4 & 2 & 13 & 50 \\
\hline Wilcoxon rank sum test & $\mathrm{P}=0.0029$ & \multicolumn{4}{|c|}{$\mathrm{P}=0.0040$} \\
\hline \multicolumn{6}{|l|}{ M Stage } \\
\hline $\mathrm{M} 0(\mathrm{n}$, mean $\pm \mathrm{SD})$ & $96,206 \pm 445$ & 12 & 5 & 27 & 57 \\
\hline M1 $(n$, mean $\pm S D)$ & $48,160 \pm 192$ & 5 & 7 & 3 & 36 \\
\hline Wilcoxon rank sum test & $\mathrm{P}=0.4582$ & \multicolumn{4}{|c|}{$\mathrm{P}=0.2523$} \\
\hline AJCC stage & 0.15 & \multicolumn{4}{|c|}{0.22} \\
\hline Spearman corr. coefficient & $\mathrm{P}=0.0588$ & \multicolumn{4}{|c|}{$\mathrm{P}=0.0046$} \\
\hline No. of positive nodes & 0.26 & \multicolumn{4}{|c|}{0.31} \\
\hline Spearman corr. coefficient & $\mathrm{P}=0.0037$ & \multicolumn{4}{|c|}{$\mathrm{P}=0.0006$} \\
\hline
\end{tabular}

Relationship between clinical factors and pathological measurements, combining all patient groups with invasive breast cancer: negative node, positive node and distant metastasis. IOD, integrated optical density; ER, estrogen receptor; PR, progesterone receptor; T-Stage, tumor stage at initial diagnosis; M-Stage, metastasis stage at initial diagnosis; M0, non known distant metastasis; M1, distant metastasis evident.

stained with an antibody recognizing CCN1. A total of 168 breast specimens were included in the analysis: 9 normal, 8 DCIS, 47 invasive node negative, 53 invasive node positive and 51 invasive with distant metastasis.

Only weak CCN1 staining could be found in normal epithelial cells. In contrast, tumor samples contained epithelial cells with prominent cytoplasmic staining (Fig. 1a). Staining intensity was assessed using both integrated OD measurements and by microscopic examination scoring from 0 to 3 . Staining levels of CCN1 were increased at all AJCC stages of disease in comparison to normal when scored by microscopic examination (Fig. 1c). The AJCC stage I grouping consists of patients with DCIS, which is characterized by proliferation of epithelial cells with no evidence of invasion through the basement membrane into the stroma. DCIS patients have an 8-10-fold greater risk of invasive breast cancer compared with women with no carcinoma. CCN1 expression was also elevated in invasive breast cancer, with scores higher than normal in AJCC stages II-IV (Fig. 1c). Moreover, the CCN1positive score correlated with the AJCC stage of disease (Spearman correlation coefficient $=0.22 ; \mathrm{P}=0.0046$ ) (Table I). Therefore, CCN1 expression can be detected at the early, proliferative stages of breast cancer, and is sustained throughout the time that the tumor invades the stroma, when lymph node involvement occurs, and when progression to distant metastasis happens.

In order to investigate the relationship between clinical factors and pathological measurements, further statistical analysis was conducted using samples from patients with invasive carcinoma (data shown in Table I). A significant correlation occurs between the CCN1 levels and the size of the tumor (Spearman correlation coefficient $=0.29$; 
(a)

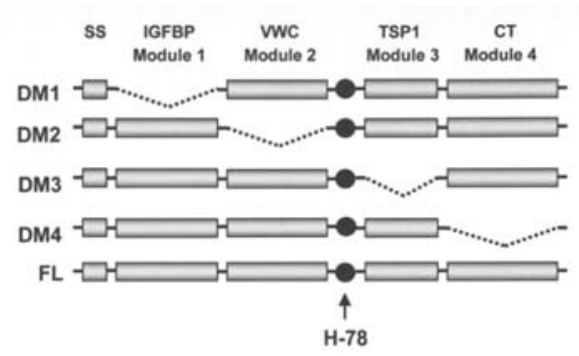

(b)

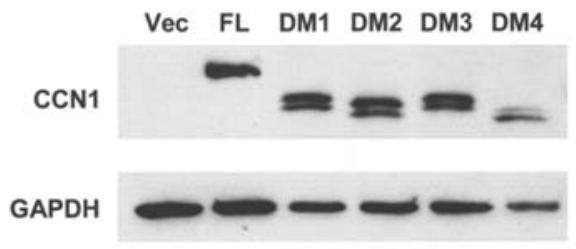

(c)

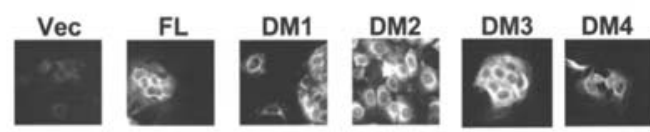

(d)

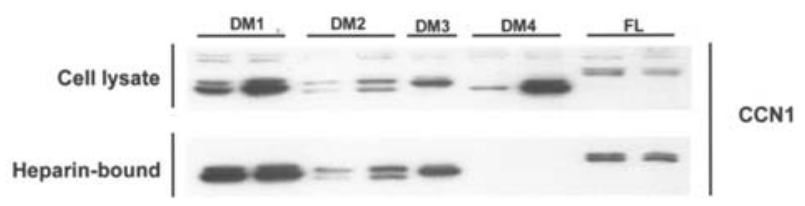

(e)

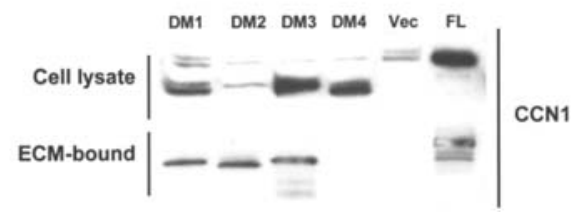

Figure 2. Deletion mutants of CCN1. (a) To investigate the contribution of each of the structural domains to the biological properties of full length (FL) CCN1 in breast cancer, mutant constructs were generated in which one of the modules (M1-M4) had been deleted. A CCN1 antibody that recognizes the middle region of the protein (H-78) could detect the presence of each of the mutants by Western blot (b) or by immunoflourescence (c). Cell lysates were incubated with heparin-agarose beads to pull down heparin-binding proteins. Proteins attached to the beads were subject to Western blotting and probed with the H-78 antibody. Module 4 of CCN1 contains two heparinbinding domains, and CCN1 could not be pulled down from protein extracts from MCF-7/DM4 cells using heparin-agarose beads (d). Extracellular matrix (ECM) produced by the cells was extracted in Laemmli sample buffer and analyzed by Western blot. The mutant CCN1 produced by MCF-7/DM4 cells was less able to associate with the ECM (e).

$\mathrm{P}=0.0011$ ). A strong association also was found between lymph node involvement and high CCN1 expression. Using Wilcoxon rank sum test, a significant increase in the CCN1positive score occurred in patients with positive lymph nodes $(\mathrm{P}=0.004)$. Furthermore, the levels of $\mathrm{CCN} 1$ correlated with the number of positive lymph nodes (Spearman correlation coefficient $=0.31 ; \mathrm{P}=0.0006)$. CCN1 levels were neither correlated with ER or PR status nor age (Table I and data not shown).

These results indicate that CCN1 expression in breast cancer is associated with an invasive phenotype and progression of the disease. Overexpression of CCN1 in breast cancer cell lines has been shown to promote their proliferation and migration in vitro and in vivo. We next conducted a structure-function analysis utilizing the modular structure of CCN1 to determine the regions of the protein that are responsible for these effects.

Expression and localization of CCN1 deletion mutants in MCF-7 cells. To investigate the contribution of each of the structural domains to the biological properties of $\mathrm{CCN} 1$ in breast cancer, we generated four mutant constructs in which one of the modules (DM1-DM4) had been deleted (Fig. 2a). MCF-7 cells were stably transfected with these constructs, and a CCN1 antibody that recognizes the middle region of the protein could detect the presence of each of the mutants by Western blot (Fig. 2b). Characteristic doublets of protein bands were detected, as previously observed by other investigators (26). Amino-truncated variants of CCN3 have been shown to localize in the nucleus of cell lines (27). However, immunofluorescence analysis showed that deletion of any of the individual modules of CCN1 did not alter the cytoplasmic localization of the protein (Fig. 2c). Module 4 of CCN1 contains 2 heparin-binding domains that are required for its heparin-binding activity. Accordingly, deletion of module 4 of CCN1 prevented pull down of CCN1 by heparinagarose beads (Fig. 2d). All mutant forms of CCN1 could be detected in the conditioned media of transfected MCF-7 cells, indicating that deletion of individual modules did not prevent their ability to follow the secretory pathway. However, module 4 was found to be important for the association of CCN1 with the ECM, as CCN1 missing this domain could not be detected in the ECM produced by MCF-7 cells (Fig. 2e).

CCN1 with deletion of module 4 is unable to promote MCF-7 cell adhesion, cell spreading, migration or proliferation. Cell motility during metastasis requires ECM attachment and cell spreading. Overexpression of full-length $\mathrm{CCN} 1$ promoted MCF-7 cell adhesion to both collagen I- and fibronectincoated culture dishes compared to control cells (Fig. 3a). However, the adhesion of MCF-7/DM4 cells to these matrix proteins was diminished; it remained at control levels for adhesion to collagen, and was reduced to below levels found with $\mathrm{MCF}-7 / \mathrm{Vec}$ cells for adhesion to fibronectin (Fig. 3a). Deletion of modules 1-3 had no effect on the ability of CCN1 to promote adhesion to either substrate.

Spreading of MCF-7/FL cells on fibronectin could clearly be seen after 30-min incubation, with the appearance of pseudopodia-like processes extending from the cells (Fig. 3b). In contrast, both MCF-7/Vec and MCF-7/DM4 cells retained a rounded morphology under these conditions. Taken together, the observations raise the possibility that $\mathrm{CCN} 1$ binding to heparan sulfate proteoglycans in the ECM is important for its ability to promote cell-ECM interactions.

Forced expression of full-length CCN1 in MCF-7 cells increases their ability to migrate $(6,23)$. We used monolayer wounding assays and Boyden chamber assays to investigate which regions of $\mathrm{CCN} 1$ are involved in promoting $\mathrm{MCF}-7$ cell migration. MCF-7/Vec cells migrated poorly in a wounding assay (Fig. 4a). Overexpression of FL-CCN1 increased the ability of MCF-7 cells to migrate across the wounded area, as 
(a)

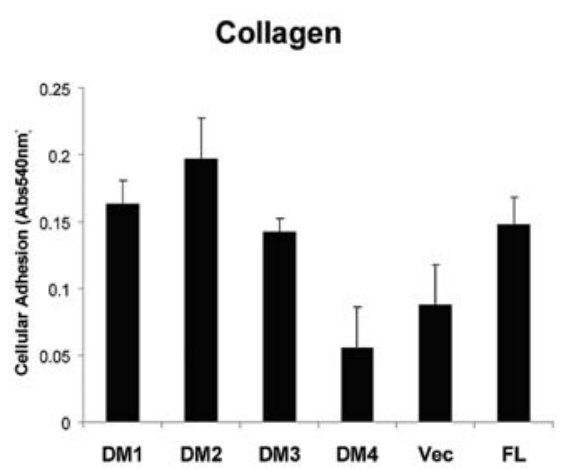

(b)

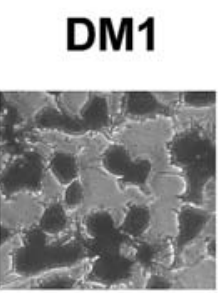

DM2

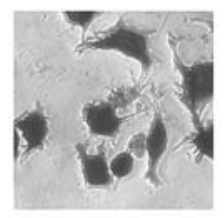

DM3

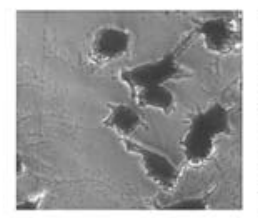

Fibronectin

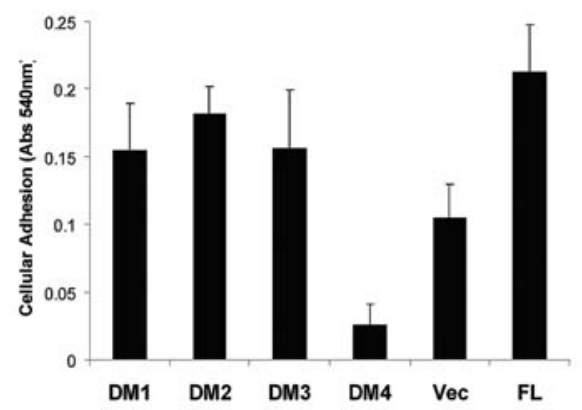

DM4

Vec

FL
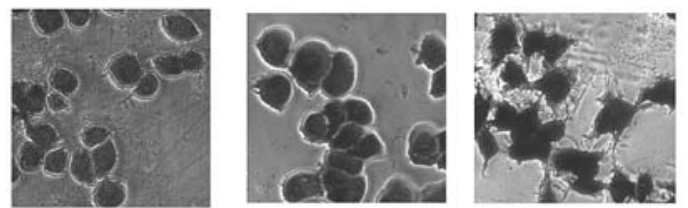

\section{Cell spreading on fibronectin}

Figure 3. Deletion of module 4 reduces the ability of CCN1 to promote MCF-7 cell spreading and attachment on the ECM. (a) Serum-starved MCF-7 cells were detached with PBS-EDTA and resuspended in media with $0.5 \%$ BSA. The mixture was added to either collagen-coated or fibronectin-coated plates and incubated for $30 \mathrm{~min}$. Attached cells were assessed by crystal violet staining. Each bar represents the mean $\pm \mathrm{SE}$ of triplicate experiments. (b) Representative phase-contrast micrographs show the morphology of serum-starved MCF-7 cells seeded onto fibronectin-coated plates for 30 min. Spreading of MCF-7/FL cells on fibronectin could clearly be seen after 30-min incubation, with the appearance of pseudopodia-like processes extending from the cells. In contrast, both MCF-7/Vec and MCF-7/DM4 cells retained a rounded morphology under these conditions. Pictures were taken at x40 magnification and are representative of two experiments.

(a)

DM1

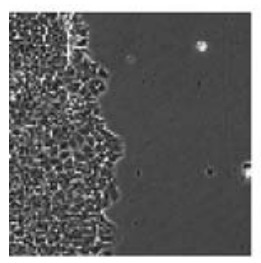

(b)
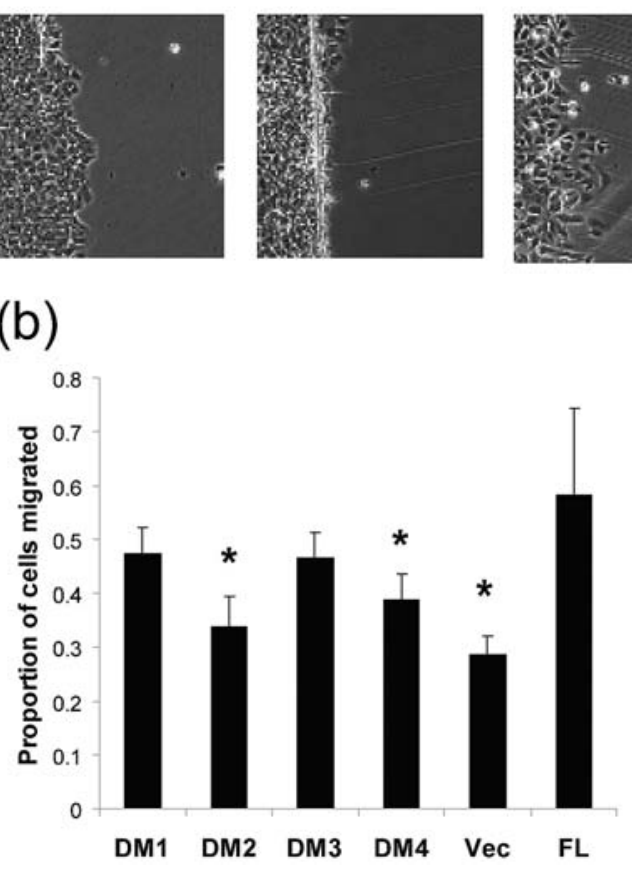

did overexpression of DM1 and DM3. In contrast, MCF-7 cells expressing DM2 and DM4 behaved similarly to control
DM4
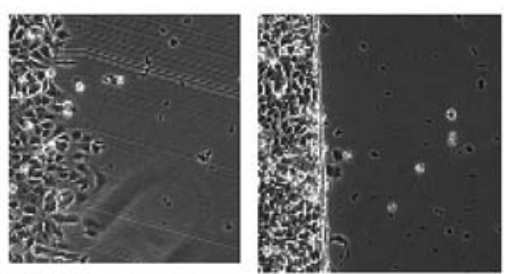

Figure 4. Deletion of modules 2 and 4 reduces the ability of CCN1 to promote pigration of MCF-7 cells. (a) Confluent layers were wounded in serum-free media and cells were monitored for their ability to migrate across the cut line. Images were raken after 24-incubation. (b) Cells $\left(2 \times 10^{5}\right)$ were placed into collagen-coated Boyden chambers. Cells were allowed to migrate for $12 \mathrm{~h}$ and the number of cells that migrated through the membrane was determined by absorbance at $540 \mathrm{~nm}$. Each bar represents the mean $\pm \mathrm{SE}$ of triplicate experiments. ${ }^{*} \mathrm{P}<0.05$ vs. MCF-7/FL.

cells. Comparable results were found using Boyden chamber assays (Fig. 4b). MCF-7/FL cells demonstrated enhanced migration in comparison to controls, and this enhancement was significantly decreased in MCF-7/DM2 and MCF-7/ DM4 cells $(\mathrm{P}<0.05)$. Taken together, these results indicate that modules 2 and module 4 of Cyr61, play key roles in its ability to promote a metastatic phenotype in breast cancer.

As reported previously, MCF-7 cells expressing fulllength $\mathrm{CCN} 1$ (MCF-7/CCN1) proliferated more rapidly than 


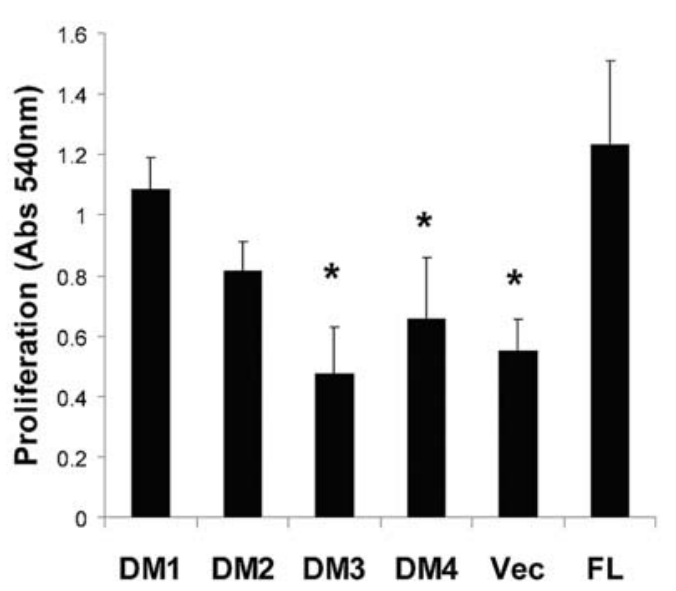

Figure 5. Proliferation of MCF-7 cells expressing deletion mutants of CCN1. As reported previously, MCF-7 cells expressing full-length CCN1 proliferated more rapidly than those expressing empty vector control cells. Deletion of either M1 or M2 had little effect on the ability of CCN1 to increase proliferation of MCF-7 cells. In contrast, the growth of MCF-7/ DM3 and MCF-7/DM4 cells was reduced to the level of MCF-7/Vec cells, suggesting a role for these regions in contributing to the growth-promoting effects of CCN1 in breast cancer. Each bar represents the mean $\pm \mathrm{SE}$ of triplicate experiments. ${ }^{*} \mathrm{P}<0.05$ vs. MCF-7/FL.

those expressing empty vector $(\mathrm{MCF}-7 / \mathrm{Vec})$ control cells $(\mathrm{P}<0.05)$. When either module 1 or module 2 was deleted, this enhanced proliferative rate in comparison to controls was maintained $(\mathrm{P}<0.05)$. In contrast, the growth of MCF-7/DM3 and MCF-7/DM4 cells was reduced to the level of MCF-7/ Vec cells, indicating a role for these regions in contributing to the growth-promoting effects of CCN1 in breast cancer.

Cyr61-transfected MCF-7 cells overexpress integrins and have increased ILK and ERK activity. An increasing number of integrins have been identified as receptors for secreted Cyr61 (28). Therefore, we examined the mRNA expression of integrin subunits in MCF-7/Vec and MCF-7/CCN1 cells using the RNAse protection assay. Expression of integrin subunits was generally higher in MCF-7/CCN1 cells compared to MCF-7/Vec cells (Fig. 6a). Interestingly, levels of integrin subunits $\alpha_{v}, \alpha_{2}, \alpha_{6}$ and $\beta_{3}$ were markedly increased when compared to controls, which is notable because of the ability of Cyr61 to interact with integrins $\alpha_{v} \beta_{3}, \alpha_{\mathrm{IIb}} \beta_{3}, \alpha_{v} \beta_{5}$ and $\alpha_{6} \beta_{1}$. ILK binds to the cytoplasmic domain of $\beta_{1}$ and $\beta_{3}$ subunits, and can promote growth in response to integrin activation. We analyzed the expression of ILK in MCF-7 cells, and determined its activity using myelin basic protein as a substrate. No difference in ILK protein levels existed between MCF-7/Vev and MCF-7/CCN1 cells (Fig. 6b). However, MCF-7/CCN1 cells possessed greater ILK activity than their control counterparts (Fig. 6b).

Integrin ligation and activation promotes cell motility, partly through up-regulation of the Ras-ERK pathway. We examined the phosphorylation status of ERK under conditions in which MCF-7/FL cells show enhanced motility (Fig. 6c). Levels of phosphorylated ERK were greatly increased in MCF-7/FL compared to MCF-7/Vec cells in both untreated and collagen-stimulated conditions. Phosphorylation of ERK was stimulated by plating MCF-7/Vec cells (a)

(b)

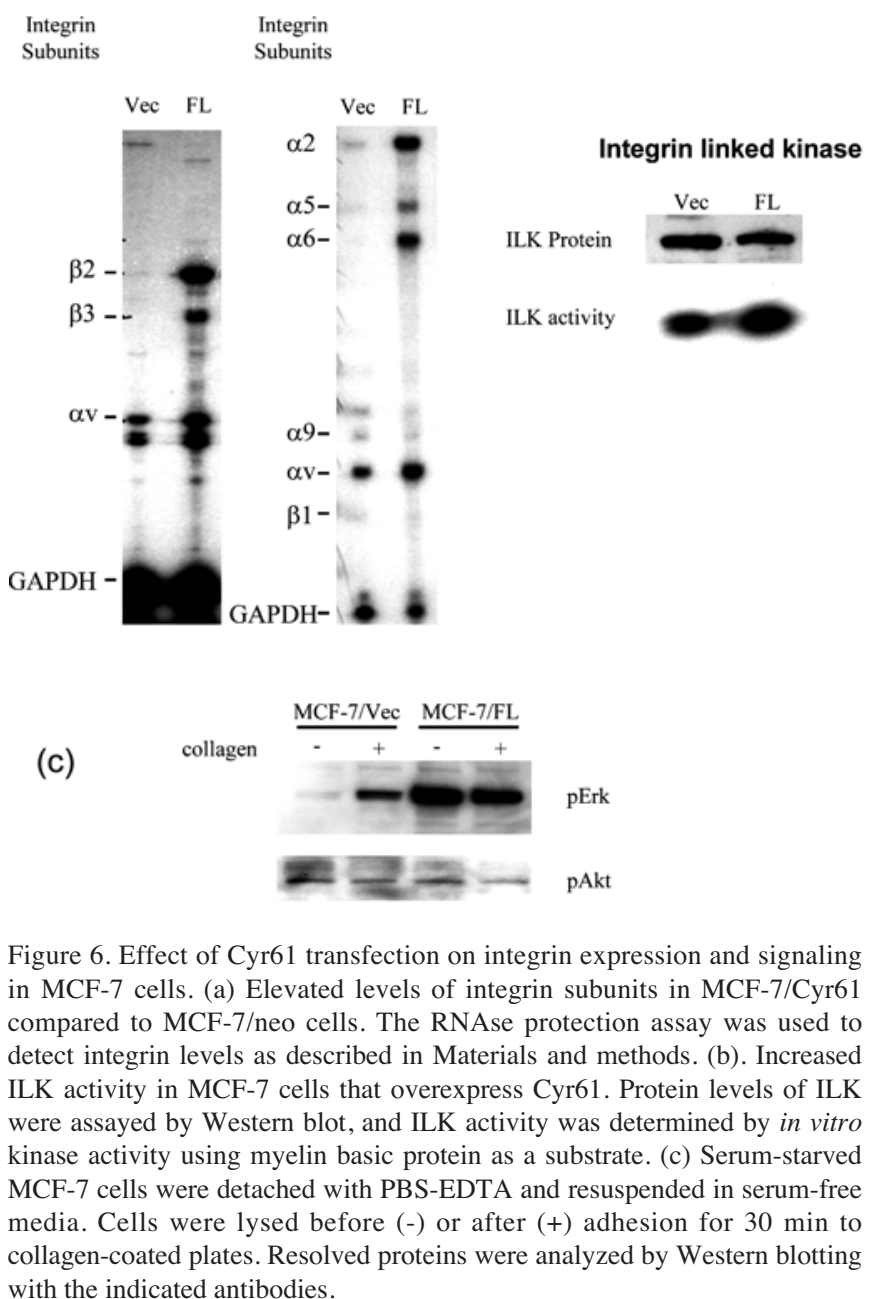

with the indicated antibodies.

onto collagen; but for MCF-7/FL cells, no further increase phosphorylated ERK occurred beyond the high levels found in these untreated cells. Differences in phosphorylation of AKT were not observed, suggesting that migration stimulated by CCN1 in MCF-7 cells is associated with specific upregulation of the ERK pathway.

\section{Discussion}

Tumor initiation and subsequent metastasis to distant sites is a multistep process controlled in part by changes occurring in the tissue microenvironment, including elevation of particular hormones and growth factors, decreased oxygen concentrations and alterations in matrix composition $(29,30)$. Many of these signals up-regulate CCN1 expression, implicating $\mathrm{CCN} 1$ at multiple stages of cancer progression. In the current study, we have demonstrated that $\mathrm{CCN} 1$ protein is found at higher levels in breast cancer samples compared to non-tumor tissue. Statistical analysis revealed a significant correlation between $\mathrm{CCN} 1$ protein levels and tumor size, stage of disease and lymph node involvement. These findings are in concordance with previous studies in which mRNA levels were analyzed $(6,22,25)$. Other investigators have demonstrated increased protein expression of CCN1 in breast 
cancer, but did not examine its relationship with clinical and pathological parameters. Our finding that elevated expression of $\mathrm{CCN} 1$ protein was detected in AJCC stage I of breast cancer suggests that it may be involved in the early phases of the transformation process. This notion is supported by our earlier work showing that overexpression of CCN1 could initiate anchorage-independent growth and in vivo xenograft formation of non-tumorigenic MCF12A breast cells (6).

$\mathrm{CCN} 1$ is an angiogenic factor that can promote cellular proliferation, survival, adhesion and migration. Therefore, it might also be expected to be involved during progression of breast cancer to a more advanced state, as demonstrated by the high expression levels found at AJCC stages 2-4. The potential involvement of clinical metastasis was further validated by the finding that $\mathrm{CCN} 1$ protein was increased in lymph node positive cancer and that increased levels of CCN1 were correlated with an increased number of positive lymph nodes. Overexpression of CCN1 in MCF-7 breast cancer cells has previously been shown to promote a metastatic phenotype in vitro and in vivo $(6,23)$. One way in which CCN1 might enhance metastasis is by up-regulation of integrin receptors and their downstream signaling pathways. Forced expression of $\mathrm{CCN} 1$ leads to increased cell surface expression of integrin $\alpha_{v} \beta_{3}(19,31)$. Here, we show that multiple integrin subunits are up-regulated in response to CCN1 overexpression, including $\alpha_{v}, \alpha_{2}, \alpha_{6}$ and $\beta_{3}$, which make up several known receptors for CCN1: integrins $\alpha_{v} \beta_{3}$, $\alpha_{\mathrm{IIb}} \beta_{3}, \alpha_{v} \beta_{5}$ and $\alpha_{6} \beta_{1}(14,13,32,33)$. Increased expression and activation of integrin receptors has been demonstrated in breast cancer and is associated with tumor metastasis $(16,34,35)$. In addition, MMTV-transgenic murine studies have shown that overexpression of the integrin signaling effector ILK in breast tissue induces mammary gland hyperplasia and tumor formation (36). The finding that CCN1 up-regulates expression of integrins and stimulates ILK and ERK activity indicates that this pathway mediates the oncogenic effects of CCN1 in breast cancer. Furthermore, it supports the hypothesis that $\mathrm{CCN} 1$ overexpression creates an autocrine signaling loop to promote tumorigenesis and cancer progression through up-regulation of its own receptors (19).

We did not find a correlation between both ER- or PRstatus and CCN1 protein expression. Conflicting reports exist regarding the relationship between CCN1 expression and ER levels. CCN1 is expressed in both ER-positive and ER-negative breast cancer specimens (6,21-23). We have previously found a correlation between CCN1 mRNA levels and ER positivity $(6,22)$. In contrast, other groups have not found a correlation in clinical samples $(21,25)$, and an inverse correlation exists between $\mathrm{CCN} 1$ expression and ER positivity in breast cancer cell lines $(6,20)$. In one study, CCN1 expression was correlated with ER positivity only when EGFR was also expressed (21) suggesting that additional factors regulate CCN1 expression in breast cancer. Indeed, CCN1 transcription is induced by multiple factors that promote breast cancer, including estrogen, EGF, hypoxia and TGFß $(6,21,37,38)$. The reason for the discrepancies found in the above studies is not clear. However, given that loss of ER status is a typical feature of breast cancer progression, and since CCN1 expression is higher in cancers with more advanced features, it is likely that the correlation between CCN1 overexpression and ER status will be restricted to certain subsets of patients.

CCN1 utilization of integrin receptors promotes the activities described in this study that are associated with breast cancer progression, namely increased cellular proliferation and motility. Binding sites for specific integrins have been identified within the different domains of CCN1, which mediate these effects in a cell-type and context-dependent manner. The CT domain contains binding sites for $\alpha_{M} \beta_{2}, \alpha_{6} \beta 1$, and HSPG $(13,14)$. Two consensus binding sites for integrin $\alpha_{6} \beta_{1}$ exist in module 4 , mediating both fibroblast adhesion and smooth muscle cell migration (12). Our finding that module 4 is required for the ability of $\mathrm{CCN} 1$ to promote cellular adhesion, spreading, and migration of MCF-7 cells is consistent with these data. The functional interaction between $\mathrm{CCN} 1$ and integrin $\alpha_{6} \beta_{1}$ is HSPG-dependent, as adhesion to $\mathrm{CCN} 1$ peptides containing these sites can be disrupted by either soluble heparin or heparinase treatment of cells. The DM4 mutant was unable to bind heparin and could not be detected in the ECM, suggesting that pro-metastatic effects of CCN1 are dependent on association with HSPG in the ECM. Defective migration was also observed when module 2 containing the VWC domain was deleted from CCN1. This region contains a binding site for integrin $\alpha_{v} \beta_{3}$ that is important for mediating adhesion and migration of endothelial cells (11).

The ability of CCN1 to stimulate proliferation of MCF-7 cells was impaired upon deletion of either of the two $\mathrm{C}$-terminal modules. This is in contrast to a previous report in which a CCN1 mutant that had the CT domain deleted was able to enhance basic fibroblast growth factor-induced DNA synthesis in fibroblasts to a degree similar to wildtype cells (33). However, in this case the mutant CCN1 did not enhance basal levels of proliferation, and many examples concerning the tissue-specific effects of CCN1 and other family members have been described in the literature (5). Accordingly, a $10-\mathrm{kDa}$ C-terminal fragment of CCN2 present in uterine secretions that contained the CT domain was found to be mitogenic for fibroblasts and smooth muscle cells (39). In addition, a C-terminal fragment of CCN2 produced by plasmin cleavage, containing both modules 3 and 4, stimulated DNA synthesis in fibroblasts (26).

These observations offer the possibility that specific regions of $\mathrm{CCN} 1$ could be targeted for therapeutic benefit to inhibit particular processes under different circumstances. Results presented here indicate that the CT domain should be targeted to prevent CCN1-mediated stimulation of proliferation and metastasis of breast cancer. Alternatively, results described elsewhere suggest that targeting the N-terminal region might inhibit angiogenesis in $\mathrm{CCN} 1$-expressing breast cancers (9). Selective inhibition of either differentiation or proliferation of cultured fibroblasts has been achieved with antibodies raised against either the $\mathrm{N}$-terminal or $\mathrm{C}$-terminal portion of CCN2, respectively, demonstrating the potential of this approach (26). Furthermore, since expression of intact CCN1 is a potential tumor suppressor in lung and endometrial cancers $(7,40)$, targeting identifiable regions of CCN1 to block pro-tumorigenic functions in a tissue-specific manner may reduce the possibility of unwanted side-effects to other organs. 


\section{Acknowledgments}

H.P. Koeffler holds the Mark Goodson Chair in Oncology Research and is a member of the Jonsson Cancer Center at UCLA. This research received support from the NIH grant RO1CA1092904 and the Womens Cancer Research Institute.

\section{References}

1. Brigstock DR: The connective tissue growth factor/cysteine rich/nephroblastoma overexpressed (CCN) family. Endocr Rev 20: 189-206, 1999.

2. Grotendorst GR, Lau LF and Perbal B: CCN proteins are distinct from and should not be considered members of the insulinlike growth factor-binding protein superfamily. Endocrinology 141: 2254-2256, 2000.

3. Babic AM, Kireeva ML, Kolesnikova TV and Lau LF: CYR61, a product of a growth factor-inducible immediate early gene, promotes angiogenesis and tumor growth. Proc Natl Acad Sci USA 95: 6355-6360, 1998

4. Kireeva ML, Mo FE, Yang GP and Lau LF: Cyr61, a product of a growth factor-inducible immediate-early gene, promotes cell proliferation, migration and adhesion. Mol Cell Biol 16: 1326-1334, 1996 .

5. O'Kelly J and Koeffler HP: The role of CCN1 in tumorigenesis and cancer progression. In: CCN proteins. Perbal B (ed). Imperial College Press, 2005.

6. Xie D, Miller CW, O'Kelly J, et al: Breast cancer: Cyr61 is overexpressed, estrogen inducible and associated with more advanced disease. J Biol Chem 276: 14187-14194, 2001.

7. Tong X, Xie D, O'Kelly J, Miller CW, Muller-Tidow C and Koeffler HP: Cyr61, a member of CCN family, is a tumor suppressor in non-small cell lung cancer. J Biol Chem 276: 47709-47714, 2001

8. Perbal B: CCN proteins: multifunctional signalling regulators. Lancet 363: 62-64, 2004.

9. Pendurthi UR, Tran TT, Post M and Rao LV: Proteolysis of CCN1 by plasmin: functional implications. Cancer Res 65: 9705-9711, 2005.

10. Bork P: The modular architecture of a new family of growth regulators related to connective tissue growth factor. FEBS Lett 327: 125-130, 1993.

11. Chen N, Leu SJ, Todorovic V, Lam SC and Lau LF: Identification of a novel integrin alphavbeta3 binding site in $\mathrm{CCN} 1$ (CYR61) critical for pro-angiogenic activities in vascular endothelial cells. J Biol Chem 279: 44166-44176, 2004.

12. Leu SJ, Chen N, Chen CC, et al: Targeted mutagenesis of the angiogenic protein CCN1 (CYR61). Selective inactivation of integrin alpha6beta1-heparan sulfate proteoglycan coreceptormediated cellular functions. J Biol Chem 279: 44177-44187, 2004.

13. Schober JM, Lau LF, Ugarova TP and Lam SC: Identification of a novel integrin $\alpha \mathrm{M} 32$ Binding Site in CCN1(CYR61), a matrcellular protein expressed in healing wounds and atherosclerotic lesions. J Biol Chem 278: 25808-25815, 2003.

14. Chen N, Chen CC and Lau LF: Adhesion of human skin fibroblasts to Cyr61 is mediated through integrin $\alpha 6 \beta 1$ and cell surface heparan sulfate proteoglycans. J Biol Chem 275: 24953-24961, 2000.

15. Taddei I, Faraldo MM, Teuliere J, Deugnier MA, Thiery JP and Glukhova MA: Integrins in mammary gland development and differentiation of mammary epithelium. J Mammary Gland Biol Neoplasia 8: 383-394, 2003.

16. Felding-Habermann B, O'Toole TE, Smith JW, et al: Integrin activation controls metastasis in human breast cancer. Proc Natl Acad Sci USA 98: 1853-1858, 2001

17. Wu C and Dedhar S: Integrin-linked linase and its interactors: a new paradigm for the coupling of extracellular matrix to actin cytoskeleton and signaling complexes. J Cell Biol 155: 505-510, 2001.

18. Vellon L, Menendez JA and Lupu R: AlphaVbeta3 integrin regulates heregulin (HRG)-induced cell proliferation and survival in breast cancer. Oncogene 24: 3759-3773, 2005.

19. Menendez JA, Vellon L, Mehmi I, Teng PK, Griggs DW and Lupu R: A novel CYR61-triggered 'CYR61-alphavbeta3 integrin loop' regulates breast cancer cell survival and chemosensitivity through activation of ERK1/ERK2 MAPK signaling pathway. Oncogene 24: 761-779, 2005.
20. Tsai MS, Hornby AE, Lakins J and Lupu R: Expression and function of CYR61, an angiogenic factor, in breast cancer cell lines and tumor biopsies. Cancer Res 60: 5603-5607, 2000.

21. Sampath D, Winneker RC and Zhang Z: Cyr61, a member of the $\mathrm{CCN}$ family, is required for $\mathrm{MCF}-7$ cell proliferation: regulation by 17 beta-estradiol and overexpression in human breast cancer. Endocrinology 142: 2540-2548, 2001.

22. Xie D, Nakachi K, Wang H, Elashoff R and Koeffler HP Elevated levels of connective tissue growth factor, WISP-1 and CYR61 in primary breast cancers associated with more advanced features. Cancer Res 61: 8917-8923, 2001.

23. Tsai MS, Bogart DF, Castaneda JM, Li P and Lupu R: Cyr61 promotes breast tumorigenesis and cancer progression. Oncogene 21: 8178-8185, 2002

24. Sampath D, Zhu Y, Winneker RC and Zhang Z: Aberrant expression of Cyr61, a member of the CCN (CTGF/Cyr61/ Cef10/NOVH) family, and dysregulation by 17 beta-estradiol and basic fibroblast growth factor in human uterine leiomyomas. J Clin Endocrinol Metab 86: 1707-1715, 2001.

25. Jiang WG, Watkins G, Fodstad O, Douglas-Jones A, Mokbel K and Mansel RE: Differential expression of the CCN family members Cyr61, CTGF and Nov in human breast cancer. Endocr Relat Cancer 11: 781-791, 2004.

26. Grotendorst GR and Duncan MR: Individual domains of connective tissue growth factor regulate fibroblast proliferation and myofibroblast differentiation. FASEB J 19: 729-738, 2005

27. Planque N, Long Li C, Saule S, Bleau AM and Perbal B: Nuclear addressing provides a clue for the transforming activity of aminotruncated CCN3 proteins. J Cell Biochem 99: 105-116, 2006

28. Lau LF and Lam SC-T: The CCN family of angiogenic regulators: The integrin connection. Exp Cell Res 248: 44-57, 1999.

29. Roskelley CD and Bissell MJ: The dominance of the microenvironment in breast and ovarian cancer. Semin Cancer Biol 12: $97-110,2002$.

30. Gupta GP and Massague J: Cancer metastasis: building a framework Cell 127: 679-695, 2006

31. Chen CC, Mo FE and Lau LF: The angiogenic factor Cyr61 activates a genetic program for wound healing in human skin fibroblasts. J Biol Chem 276: 47329-47337, 2001

32. Jedsadayanmata A, Chen CC, Kireeva ML, Lau LF and Lam SC: Activation-dependent adhesion of human platelets to Cyr61 and Fisp12/mouse connective tissue growth factor is mediated through integrin alpha(IIb)beta(3). J Biol Chem 274: 24321-24327, 1999

33. Grzeszkiewicz TM, Kirschling DJ, Chen N and Lau LF: CYR61 stimulates human skin fibroblast migration through Integrin alpha $\mathrm{v}$ beta 5 and enhances mitogenesis through integrin alpha $\mathrm{v}$ beta 3 , independent of its carboxyl-terminal domain. J Biol Chem 276: 21943-21950, 2001.

34. Liapis $\mathrm{H}$, Flath $\mathrm{A}$ and Kitazawa $\mathrm{S}$ : Integrin alpha V beta 3 expression by bone-residing breast cancer metastases. Diagn Mol Pathol 5: 127-135, 1996

35. Pecheur I, Peyruchaud O, Serre CM, et al: Integrin alpha(v) beta3 expression confers on tumor cells a greater propensity to metastasize to bone. FASEB J 16: 1266-1238, 2002.

36. White DE, Cardiff RD, Dedhar S and Muller WJ: Mammary epithelial-specific expression of the integrin-linked kinase (ILK) results in the induction of mammary gland hyperplasias and tumors in transgenic mice. Oncogene 20: 7064-7072, 2001.

37. Kunz M, Moeller S, Koczan D, et al: Mechanisms of hypoxic gene regulation of angiogenesis factor Cyr61 in melanoma cells. J Biol Chem 278: 45651-45660, 2003.

38. Bartholin L, Wessner LL, Chirgwin JM and Guise TA: The human Cyr61 gene is a transcriptional target of transforming growth factor beta in cancer cells. Cancer Lett 246: 230-236, 2007.

39. Brigstock DR, Steffen CL, Kim GY, Vegunta RK, Diehl JR and Harding PA: Purification and characterization of novel heparinbinding growth factors in uterine secretory fluids. Identification as heparin-regulated $\mathrm{Mr} \mathrm{10,000} \mathrm{forms} \mathrm{of} \mathrm{connective} \mathrm{tissue} \mathrm{growth}$ factor. J Biol Chem 272: 20275-20282, 1997

40. Chien W, Kumagai T, Miller CW, et al: Cyr61 suppresses growth of human endometrial cancer cells. J Biol Chem 279: 53087-53096, 2004. 